\title{
On the Convergence of Difference Approximations to Nonlinear Contraction Semigroups in Hilbert Spaces
}

\author{
By Olavi Nevanlinna*
}

Abstract. Convergence properties of the difference schemes

$$
h^{-1} \sum_{j=0}^{k} \alpha_{j} u_{n+j}+\sum_{j=0}^{k} \beta_{j} A u_{n+j}=0, \quad n \geqslant 0,
$$

for evolution equations

$$
\frac{d u(t)}{d t}+A u(t)=0, \quad t \geqslant 0 ; \quad u(0)=u_{0} \in \overline{D(A)}
$$

\begin{abstract}
are studied. Here $A$ is a nonlinear, maximally monotone operator in a real Hilbert space. It is shown, in particular, that if the scheme (S) is consistent and stable for the test equation $x^{\prime}=\lambda x$ for $\lambda \in \mathrm{C}-K$, where $K$ is a compact subset of the right half-plane, then (S) is convergent as $h \downarrow 0$, with suitable initial values, for (E), on compact intervals $[0, T]$. Moreover, the convergence is uniform on the half-axis $t \geqslant 0$, if the solution $u(t)$ tends strongly to a constant as $t \rightarrow \infty$. We also show that under weaker stability conditions one can construct conditionally convergent methods.
\end{abstract}

Introduction. We study the approximation of nonlinear contraction semigroups by means of linear multistep methods. In an earlier paper [12] we derived bounds for the accumulated errors in terms of the local (truncation) errors. If the solutions are continuously differentiable, then the local errors are small enough for the error bounds to imply the convergence as the step length tends to zero, uniformly on compact time intervals.

Here we study the convergence without assuming smoothness of the solutions. In order to show the convergence on compact time intervals we replace the operator by its Yosida approximation. Since the Yosida approximation is Lipschitz-continuous, the solutions are in $C^{1}$ and our difference methods yield convergent approximations. The main task then is to bound the difference between the solutions of the original and approximated difference equations.

For one special method, namely the implicit Euler method, the convergence on compact time intervals is well known, stating that the semigroup $S(t)$ is obtained from the generator $-A$ by the product formula

$$
S(t) x=\lim _{n \rightarrow \infty}\left(I+\frac{t}{n} A\right)^{-n} x \text { for all } x \in \overline{D(A)},
$$

Received May 31, 1977.

AMS (MOS) subject classifications (1970). Primary 65J05.

* This work was done during the author's stay at IBM Research Center, Yorktown Heights, New York 10598. 
see [1], [2]. Difficulties in generalizing this result to other methods are hidden in the fact that the contractiveness is generally not preserved under discretization. In Hilbert spaces we still have enough contraction-like behavior to proceed (see Theorem 1 below), but in Banach spaces the only results we know are for the implicit Euler method, see, e.g. [4] , [9], [13].

Under some conditions $S(t) x$ tends strongly to a limit as $t \rightarrow \infty$, see [3]. We show that our approximations then converge not only on compact time intervals but uniformly on the half-axis $t \geqslant 0$.

We also demonstrate that our class of methods cannot substantially be enlarged, and give a convergence rate for problems with initial values inside the domain of the operator $A$.

We finally show that all stable multistep methods which are not weakly stable can be used to build conditionally convergent methods, where one approximates the nonlinear operator by its Yosida approximation and relates the growth of the Lipschitz constant to the decrease of the step length.

2. Results. Let $H$ be a real Hilbert space and $A$ a generally nonlinear singlevalued operator $D(A) \subset H \rightarrow H$. The operator $A$ is said to be monotone if

$$
(u-v, A u-A v) \geqslant 0 \quad \text { for all } u, v \in D(A),
$$

and maximally monotone if additionally $R(I+A)=H$. By the solution of the initial value problem

$$
\frac{d u(t)}{d t}+A u(t)=0, \quad t \geqslant 0, \quad u(0)=u_{0} \in \overline{D(A)}
$$

we shall mean the unique continuous function $u: t \rightarrow S(t) u_{0}$, where $S(t)$ denotes the contraction semigroup generated by $-A$, see [1]. The initial value problem is approximated using linear multistep methods $(\rho, \sigma)$ :

$$
h^{-1} \rho(E) u_{n}^{h}+\sigma(E) A u_{n}^{h}=0, \quad n \geqslant 0, \quad u_{0}^{h}, \ldots, u_{k-1}^{h} \in D(A) \text { given. }
$$

In (3) $E$ denotes the translation operator $E y_{n}=y_{n+1}, h>0$ is the step length and $\rho$ and $\sigma$ are polynomials

$$
\rho(\zeta)=\sum_{j=0}^{k} \alpha_{j} \xi^{j}, \quad \sigma(\zeta)=\sum_{j=0}^{k} \beta_{j} \xi^{j}
$$

with real coefficients and with no common divisor.

We first give a result concerning the solutions of (3).

Consider

$$
h^{-1} \rho(E) u_{n}+\sigma(E) A u_{n}=r_{n+k}, \quad n \geqslant 0,
$$




$$
h^{-1} \rho(E)\left(u_{n}-v_{n}\right)+\sigma(E)\left(A u_{n}-A v_{n}\right)=f_{n+k}, \quad n \geqslant 0 .
$$

THEOREM 1. Suppose that the method $(\rho, \sigma)$ satisfies

$$
\begin{gathered}
\rho(1)=0, \quad \rho^{\prime}(1)=\sigma(1), \\
\operatorname{Re} \frac{\rho}{\sigma}(\zeta)>0 \quad \text { for }|\zeta|>1, \\
\sigma(\zeta) \neq 0 \quad \text { on }|\zeta|=1 .
\end{gathered}
$$

If $A$ is maximally monotone, then for any given $\left\{r_{n}\right\} \subset H$ and $\left\{u_{0}, \ldots, u_{k-1}\right\} \subset$ $D(A)$ there exists a unique $\left\{u_{n}\right\} \subset D(A)$ satisfying (5). Moreover, there exists a constant $C$, depending only on the method $(\rho, \sigma)$ such that for $\left\{u_{n}-v_{n}\right\}$ satisfying (6) we have, for all $n \geqslant k$,

$$
\left|u_{n}-v_{n}\right| \leqslant C\left\{\max _{0 \leqslant j<k}\left[\left|u_{j}-v_{j}\right|+h\left|A u_{j}-A v_{j}\right|\right]+h \sum_{j=k}^{n}\left|f_{j}\right|\right\}
$$

This result is included in Theorems 2.1 and 3.1 of [12]. We first observe that if we can show convergence for some special initial values $u_{0}^{h}, \ldots, u_{k-1}^{h}$ in the absence of round-off errors $\left(r_{n}=0\right)$, then the general case can be obtained from (10).

Theorem 2. Assume that (7), (8) and (9) hold. Let A be maximally monotone and $u$ the solution of (2). Set $u_{j}^{h}=(I+h A)^{-1} u_{0}$ for $j=0, \ldots, k-1$, and let $\left\{u_{n}^{h}\right\}$ satisfy (3). Then, for all $T<\infty$,

$$
\sup _{0 \leqslant n<T h^{-1}}\left|u(n h)-u_{n}^{h}\right| \rightarrow 0 \quad \text { as } h \rightarrow 0 .
$$

The proof of Theorem 2 will give us the following additional result.

Corollary 1. If, in addition to the assumptions of Theorem 2, $u_{0} \in D(A)$, then for all $T<\infty, h<\infty$

$$
\sup _{0 \leqslant n<T / h}\left|u(n h)-u_{n}^{h}\right| \leqslant C\left|A u_{0}\right|\left\{h+T^{1 / 2} h^{1 / 2}+\left[T+T^{1 / 2}\right] h^{1 / 3}\right\},
$$

with $C$ depending only on the method $(\rho, \sigma)$.

For the implicit Euler method ("Rothe method"), applied to a class of nonlinear initial boundary value problems Kačur [8] has obtained a convergence order $O\left(h^{1 / 2}\right)$. Recall that if the solutions are in $C^{1}$, then Theorem 1 implies the convergence order $O(h)$.

The next example shows that the assumption $u_{0} \in D(A)$ in Corollary 1 is essential for getting the convergence order $O\left(h^{1 / 3}\right)$. Let $H=\mathbf{R}$ and put $A u=-u^{1-s}$ for $u>0$ and $s>1$. Then $u(t)=s^{1 / s} t^{1 / s}$ is the solution of $u^{\prime}+A u=0$ with $u(0)$ $=0$. But the implicit Euler method,

$$
h^{-1}\left(u_{n+1}-u_{n}\right)-\left(u_{n+1}\right)^{1-s}=0,
$$


with the exact initial value $u_{0}=0$ yields $u_{1}=h^{1 / s}$. Hence $\left|u(h)-u_{1}\right|=\left(s^{1 / s}-1\right) h^{1 / s}$ for the exact initial value, and for the one of Theorem $2, u_{0}^{h}=(I+h A)^{-1} 0=h^{1 / s}$, we have $\left|u(0)-u_{0}^{h}\right|=h^{1 / s}$.

THEOREM 3. If in addition to the assumptions of Theorem 2

$$
u(t) \rightarrow c \text { as } t \rightarrow \infty,
$$

then

$$
\sup _{n \geqslant 0}\left|u(n h)-u_{n}^{h}\right| \rightarrow 0 \quad \text { as } h \rightarrow 0
$$

It is shown in [3] that if $A=\partial \varphi$ and $\varphi(-u)=\varphi(u)$ for all $u$ then (13) holds. Another sufficient condition is that $0 \in R(\partial \varphi)$ and the set $\{u \in H|| u \mid \leqslant M, \varphi(u) \leqslant M\}$ is compact for all $M<\infty[1, \mathrm{p} .90]$. Finally, note that if $A-\alpha I$ is monotone for some $\alpha>0$, then $u(t)$ tends exponentially to the unique solution of $0=A c$.

Consider the assumptions on $(\rho, \sigma)$. The (algebraic) consistency conditions (7) are certainly necessary for the convergence (11). We shall now demonstrate that also the assumption (8) is necessary. Methods satisfying (8) are called $A$-stable [5]. If (8) does not hold, then there exists a $q \in \mathbf{C}$ with $\operatorname{Re} q<0$ such that the equation

$$
\rho(\zeta)-q \sigma(\zeta)=0
$$

has at least one solution $\zeta=\xi$ satisfying $|\xi|>1$. Hence, the difference equation

$$
\rho(E) \eta_{n}=\lambda h \sigma(E) \eta_{n}
$$

has, for $\lambda h=q$ and for initial values $\eta_{j}=\xi^{j} x, j=0, \ldots, k-1$, the solution $\eta_{n}=$ $\xi^{n} x$. Let $l^{2}$ be the (complex) Hilbert space of sequences $x=\left(x^{\mu}\right)_{\mu>0}$, with $x^{\mu} \in \mathbf{C}$ and $(x, y)=\Sigma_{\mu \geqslant 0} \bar{y}^{\mu} x^{\mu}$, and let $\Lambda$ be the operator mapping $\left(x^{\mu}\right)$ to $\left(\lambda_{\mu} x^{\mu}\right)$. Then the solutions of

$$
h^{-1} \rho(E) x_{n}=\sigma(E) \Lambda x_{n}
$$

satisfy

$$
\rho(E) x_{n}^{\mu}=h \lambda_{\mu} \sigma(E) x_{n}^{\mu}
$$

Put $x_{0}=\left(\mu^{-\alpha}\right)$ with $\alpha>2$ and choose $\lambda_{\mu}=q \mu$. Then $\Lambda x_{0} \in l^{2}$ and hence $x_{0} \in$ $D(\Lambda)$. Now choose a sequence $h_{\mu}$ tending to zero by setting $h_{\mu}=\mu^{-1}$. Then, for all $n$

$$
\left\|x_{n}\right\|_{2} \geqslant\left\|x_{n}\right\|_{\infty} \geqslant|\xi|^{n}\left|x_{0}^{\mu}\right|
$$

But, as $h_{\mu} \rightarrow 0,|\xi|^{T h_{\mu}^{-1}}\left|x_{0}^{\mu}\right|=\left(|\xi|^{T}\right)^{\mu} \mu^{-\alpha} \rightarrow \infty$ and therefore,

$$
\left\|x_{T h_{\mu}^{-1}}-x(T)\right\|_{2} \rightarrow \infty \quad \text { as } h_{\mu} \downarrow 0
$$


The assumption (9), on the contrary, seems to be a technical one. A practical method excluded by (9) is the trapezoidal rule

$$
h^{-1}\left(u_{n+1}^{h}-u_{n}^{h}\right)+1 / 2\left(A u_{n+1}^{h}+A u_{n}^{h}\right)=0, \quad n \geqslant 0 .
$$

THEOREM 4. Let $A$ be maximally monotone and $u$ the solution of (2). Set $u_{0}^{h}=(I+h A / 2)^{-1} u_{0}$, then for all $h>0$ a unique $\left\{u_{n}^{h}\right\} \subset D(A)$ exists satisfying (15), and for all $T<\infty$ (11) holds. Moreover, we have (14) whenever (13) holds.

It is natural to ask whether we could relax (9) by approximating the unbounded operator $A$ by a Lipschitz-continuous operator and obtain conditionally convergent methods. In order to state a result of this type we introduce some notation. If $A$ is maximally monotone, then the operator

$$
J_{\lambda}=(I+\lambda A)^{-1}, \quad \lambda>0
$$

is a contraction defined for all $x \in H$; and therefore, the operator

$$
A_{\lambda}=\lambda^{-1}\left(I-J_{\lambda}\right)
$$

referred to as the Yosida approximation of $A$, is Lipschitz-continuous. We shall also need the concept of stability region of a method $(\rho, \sigma)$ which is defined to be the set $S$ of complex numbers $q$ for which the characteristic polynomial $\rho(\zeta)-q \sigma(\zeta)$ satisfies the root condition, i.e. its roots satisfy $\left|\zeta_{\nu}\right| \leqslant 1$ and those of modulus one are simple. Our stability assumption on the method $(\rho, \sigma)$ is

$$
\text { there exists } r>0 \text { such that }\{z \in \mathbf{C}|| z+r \mid \leqslant r\} \subset S \text {. }
$$

It can be shown that $(16)$ holds for all stable methods $(\rho, \sigma)$ which are not "weakly stable" (defined in terms of the growth parameters; G. Dahlquist, personal communication).

We shall approximate the solution of (2) by the scheme

$$
h^{-1} \rho(E) u_{\lambda, n}^{h}+\sigma(E) A_{\lambda} u_{\lambda, n}^{h}=0, \quad n \geqslant 0 .
$$

Note that since $A_{\lambda}$ is Lipschitz-continuous with the constant $1 / \lambda$, (17) has a unique solution $\left\{u_{\lambda, n}^{h}\right\} \subset H$, whenever $h / \lambda$ is small enough, for all given $\left\{u_{\lambda, 0}^{h}, \ldots, u_{\lambda, k-1}^{h}\right\}$ $\subset H$.

Theorem 5. Suppose that the method $(\rho, \sigma)$ satisfies (7) and (16). Let $A$ be maximally monotone and $u$ the solution of $(2)$, with $u_{0} \in D(A)$. Set $u_{\lambda, j}^{h}=u_{0}$ for $j=0, \ldots, k-1$, and suppose $\left\{u_{\lambda, n}^{h}\right\}$ satisfies (17). Assume that for a given $c>0$, we have $\lambda^{2} \geqslant c h$. Then there exist constants $\lambda_{0}=\lambda_{0}(\rho, \sigma, c)>0$ and $C=$ $C(\rho, \sigma, T, c)$ such that if $\lambda \in\left(0, \lambda_{0}\right)$, then

$$
\sup _{0 \leqslant n<T / h}\left|u(n h)-u_{\lambda, n}^{h \cdot}\right| \leqslant C\left|A u_{0}\right|\left\{(h / \lambda)+\lambda^{1 / 2}\right\} .
$$

We finally point out that (16) cannot be replaced by just assuming that $\rho$ is stable, that is $0 \in S$. Consider the "leap-frog" method $(\rho, \sigma)=\left(\zeta^{2}-1,2 \zeta\right)$ for which 
$S$ is the open interval $(-i, i)$. This method can be used as a basis for conditionally stable schemes for some linear hyperbolic equations. Let $H$ be the real Hilbert space $l^{2}$ of square summable sequences $\left(x^{\mu}\right)_{\mu \geqslant 0}$ and let $A$ be the linear operator $\left(x^{\mu}\right) \rightarrow$ $\left(\alpha^{\mu} x^{\mu}\right)$ with $\alpha>1$. Hence $A_{\lambda}$ is the operator $\left(x^{\mu}\right) \rightarrow\left(\alpha^{\mu}\left(1+\lambda \alpha^{\mu}\right)^{-1} x^{\mu}\right)$. It is easily seen that there exist initial values $x_{0}, x_{1}$, depending on $\lambda$ and $h$ but uniformly bounded, such that $\left\|x_{T / h}\right\|_{2} \rightarrow \infty$ as $\lambda, h \rightarrow 0$.

3. Proof of Theorem 2. We first show that if the Theorem 2 is true for all $u_{0} \in D(A)$, the general case will follow. Assume, therefore, that $u_{0} \in \overline{D(A)}$ and fix $\epsilon>0$. Pick a $v_{0} \in D(A)$ so that $\left|u_{0}-v_{0}\right|<\epsilon$. Then due to the contraction property of the solutions, we have

$$
|u(t)-v(t)| \leqslant\left|u_{0}-v_{0}\right|<\epsilon \text { for } t \geqslant 0
$$

For $\left|u_{n}^{h}-v_{n}^{h}\right|$ we get from Theorem 1

$$
\left|u_{n}^{h}-v_{n}^{h}\right| \leqslant C\left\{\left|u_{0}^{h}-v_{0}^{h}\right|+h\left|A u_{0}^{h}-A v_{0}^{h}\right|\right\} \quad \text { for } n \geqslant 0, h>0
$$

( $C$ will denote a constant which is independent on $n, h, \lambda, T$ but not necessarily the same at each occurrence.)

We recall some well-known properties of the resolvent $J_{\lambda}=(I+\lambda A)^{-1}$ and the Yosida approximation $A_{\lambda}=(1 / \lambda)\left(I-J_{\lambda}\right)$, of a maximally monotone operator $A$ :

(i) $\left|A_{\lambda} x\right| \uparrow|A x|$ and $A_{\lambda} x \rightarrow A x$ for $x \in D(A)$ as $\lambda \downarrow 0$,

(ii) $\left|A_{\lambda} x\right| \uparrow \infty$ for $x \notin D(A)$ as $\lambda \downarrow 0$,

(iii) $A_{\lambda} x=A J_{\lambda} x$ for all $x \in H, \lambda>0$.

Since $J_{\lambda}$ is a contraction for $\lambda>0, A_{\lambda}$ is (maximally monotone, and) Lipschitz-continuous with constant $1 / \lambda$.

Using (iii), we get

$$
h\left|A u_{0}^{h}-A v_{0}^{h}\right|=h\left|A J_{h} u_{0}-A J_{h} v_{0}\right|=h\left|A_{h} u_{0}-A_{h} v_{0}\right| \leqslant\left|u_{0}-v_{0}\right|<\epsilon .
$$

Substituting this into (20) yields

$$
\left|u_{n}^{h}-v_{n}^{h}\right| \leqslant 2 C \epsilon \quad \text { for all } n \geqslant 0, h>0
$$

Suppose now that Theorem 2 holds for all $v_{0} \in D(A)$ so that

$$
\sup _{0 \leqslant n<T / h}\left|v(n h)-v_{n}^{h}\right| \rightarrow 0 \quad \text { as } h \downarrow 0 .
$$

Fix $h_{0}$ so that

$$
\left|v(n h)-v_{n}^{h}\right|<\epsilon \quad \text { for } 0<h<h_{0}, 0 \leqslant n<T / h \text {. }
$$

Then, for $0<h<h_{0}$, 


$$
\begin{aligned}
\sup _{0 \leqslant n<T / h}\left|u(n h)-u_{n}^{h}\right| & \\
& \leqslant|u(n h)-v(n h)|+\left|v(n h)-v_{n}^{h}\right|+\left|v_{n}^{h}-u_{n}^{h}\right| \leqslant \epsilon+\epsilon+2 C \epsilon,
\end{aligned}
$$

which completes the reduction.

From now on we assume that $u_{0} \in D(A)$. We intend to use the known convergence for Lipschitz-continuous problems. The latter is well known; it also follows easily from (10), with $u_{n}=u_{n}^{h}, v_{n}=u(n h)$, and with $\left\{q_{n}\right\}=\left\{\tau_{n}^{h}\right\}$ representing the local discretization errors which are sufficiently small because solutions to Lipschitzcontinuous problems are continuously differentiable. In fact, replace $A$ by $A_{\lambda}$ and denote the solutions of the differential and the difference equation by $u_{\lambda}(t)$ and $u_{\lambda, n}^{h}$, respectively. Then $\left\{\tau_{\lambda, n}^{h}\right\}$ is defined by

$$
h^{-1} \rho(E) u_{\lambda}(n h)+\sigma(E) A_{\lambda} u_{\lambda}(n h)=\tau_{\lambda, n}^{h} \text { for } n \geqslant 0, h, \lambda>0
$$

Using the mean-value theorem and the fact that $\left|A_{\lambda} u_{\lambda}(t)\right| \leqslant\left|A_{\lambda} u_{\lambda}(0)\right|$ (see Theorem 3.1 in [1]), we have

$$
\left|\tau_{\lambda, n}^{h}\right| \leqslant C(h / \lambda)\left|A_{\lambda} u_{\lambda}(0)\right|
$$

with $C$ depending only on $(\rho, \sigma)$. We shall put $u_{\lambda}(0)=u_{0}$ and $u_{\lambda, j}^{h}=u_{0}^{h}$ for $j=$ $0, \ldots, k-1$. Then $(10)$ gives us

$$
\left|u_{\lambda}(n h)-u_{\lambda, n}^{h}\right| \leqslant C\left\{\left|u_{0}-u_{0}^{h}\right|+h\left|A_{\lambda} u_{0}-A_{\lambda} u_{0}^{h}\right|+h \sum_{j=k}^{n}\left|\tau_{\lambda, j}^{h}\right|\right\} .
$$

But $\left|u_{0}-u_{0}^{h}\right|=\left|u_{0}-J_{h} u_{0}\right|=h\left|A u_{0}\right|$ and $h\left|A_{\lambda} u_{0}-A_{\lambda} u_{0}^{h}\right| \leqslant 2 h\left|A u_{0}\right|$, so that (23) and (24) yield

$$
\left|u_{\lambda}(n h)-u_{\lambda, n}^{h}\right| \leqslant C\{1+(T / \lambda)\}\left|A u_{0}\right| h, \quad 0 \leqslant n<T / h .
$$

For $\left|u(t)-u_{\lambda}(t)\right|$ the following bound is given in $[1$, p. 56]:

$$
\left|u(t)-u_{\lambda}(t)\right| \leqslant(1 / \sqrt{2})\left|A u_{0}\right| \sqrt{ }(\lambda t), \quad t \geqslant 0 .
$$

In order to obtain the convergence of $u_{n}^{h}$ we also need a bound for $\left|u_{n}^{h}-u_{\lambda, n}^{h}\right|$. We start with the equation

$$
h^{-1} \rho(E)\left(u_{n}^{h}-u_{\lambda, n}^{h}\right)+\sigma(E)\left(A u_{n}^{h}-A_{\lambda} u_{\lambda, n}^{h}\right)=0, \quad n \geqslant 0 .
$$

Define $u_{\lambda, n}^{h}=u_{n}^{h}=u_{0}^{h}$ for $n<0$, and denote $-A u_{n}^{h}+A_{\lambda} u_{\lambda, n}^{h}=z_{n}$. Then we have for all $n \in \mathbf{Z}$

$$
h^{-1} \rho(E)\left(u_{n}^{h}-u_{\lambda, n}^{h}\right)=p_{n},
$$

with $p_{n}=0$ for $n<0$ and $p_{n}=\sigma(E) z_{n}$ for $n \geqslant 0$. 
By assumption $(9), \sigma(\zeta)^{-1} \rho(\zeta)$ is analytic in some neighborhood of the unit circle and has an expansion $\sigma(\zeta)^{-1} \rho(\zeta) \sim \gamma_{0}+\gamma_{1} \zeta^{-1}+\gamma_{2} \zeta^{-2}+\cdots$ with $\gamma=\left\{\gamma_{n}\right\} \in$ $l^{1}$. Hence, (28) implies

$$
h^{-1} \sum_{j=0}^{n} \gamma_{n-j}\left(u_{j}^{h}-u_{\lambda, j}^{h}\right)=w_{n} \text { for } n \geqslant 0
$$

where $w_{n}$ is obtained by solving

$$
\begin{array}{ll}
\sigma(E) w_{n}=\sigma(E) z_{n} & \text { for } n \geqslant 0, \\
w_{n}=0 & \text { for } n<0, \\
\sigma(E) w_{n}=0 & \text { for } n<0 .
\end{array}
$$

Hence, $w_{n}=z_{n}+r_{n}$, where

$$
z_{n}=-A u_{n}^{h}+A_{\lambda} u_{\lambda, n}^{h}
$$

and

$$
\left|r_{n}\right| \leqslant C r^{n} \max _{0 \leqslant j<k}\left|z_{j}\right| \quad \text { with some } r<1
$$

so that

$$
\sum_{0}^{\infty}\left|r_{n}\right| \leqslant C\left|A u_{0}^{h}-A_{\lambda} u_{0}^{h}\right| \leqslant 2 C\left|A u_{0}\right|
$$

Multiplying (29) by $h\left(u_{n}^{h}-u_{\lambda, n}^{h}\right)$ and summing yields

$$
\begin{aligned}
\sum_{n=0}^{N}\left(u_{n}^{h}-\right. & \left.u_{\lambda, n}^{h}, \sum_{j=0}^{n} \gamma_{n-j}\left(u_{j}^{h}-u_{\lambda, j}^{h}\right)\right) \\
& =-h \sum_{n=0}^{N}\left(u_{n}^{h}-u_{\lambda, n}^{h}, A u_{n}^{h}-A_{\lambda} u_{\lambda, n}^{h}\right)+h \sum_{n=0}^{N}\left(u_{n}^{h}-u_{\lambda, n}^{h}, r_{n}\right) .
\end{aligned}
$$

It is shown in the proof of Theorem 3.1 in [12], that the left-hand side of (30) can be bounded from below by $\delta\left|u_{N}^{h}-u_{\lambda, N}^{h}\right|^{2}$, with $\delta>0$ depending only on the method $(\rho, \sigma)$. We shall consider the right-hand side of (30). Put $v_{n} \equiv u_{0}^{h}$ and use (10). For $n<T h^{-1}$ we then get

$$
\left|u_{\lambda, n}^{h}-u_{0}^{h}\right| \leqslant C h \sum_{j=k}^{n}\left|A_{\lambda} u_{0}^{h}\right| \leqslant C T\left|A u_{0}\right|
$$

where we also used (i) and (iii). Similarly, we get the uniform boundedness for $\left\{u_{n}^{h}\right\}$. Hence, 


$$
h \sum_{n=0}^{N}\left(u_{n}^{h}-u_{\lambda, n}^{h}, r_{n}\right) \leqslant C T\left|A u_{0}\right|^{2} h, \quad N<T h^{-1} .
$$

To bound the first term in the right-hand side of (30) write $J_{\lambda} x=x-\lambda A_{\lambda} x$. Since $A$ is monotone, we have $\left(A J_{\lambda} x-A y, J_{\lambda} x-y\right) \geqslant 0$; and using also $A_{\lambda} x=A J_{\lambda} x$, we get

$$
\left(A_{\lambda} x-A y, x-y\right) \geqslant \lambda\left|A_{\lambda} x\right|^{2}-\lambda\left(A y, A_{\lambda} x\right) \geqslant-\frac{\lambda}{2}|A y|^{2} .
$$

Hence,

$$
h \sum_{0}^{N}\left(u_{n}^{h}-u_{\lambda, n}^{h}, A u_{n}^{h}-A_{\lambda} u_{\lambda, n}^{h}\right) \geqslant-\frac{\lambda}{2} h \sum_{n=0}^{N}\left|A u_{n}^{h}\right|^{2}
$$

We show that

$$
\left|A u_{n}^{h}\right| \leqslant C\left|A u_{0}\right|, \quad n \geqslant 0, h>0 .
$$

Apply (10) with $u_{n}=u_{n}^{h}$ and $v_{n}=u_{n+1}^{h}$. Then

$$
\left|u_{n+1}^{h}-u_{n}^{h}\right| \leqslant C\left\{\left|u_{k}^{h}-u_{0}^{h}\right|+h\left|A u_{k}^{h}-A u_{0}^{h}\right|\right\} \quad \text { for all } n \geqslant 0 .
$$

Suppose we normalize: $\alpha_{k}=1$ and put $\beta_{k}=\beta$. Then $u_{k}^{h}$ is given by

$$
u_{k}^{h}+h \beta_{k} A u_{k}^{h}=u_{0}^{h}-h\left(\sigma(1)-\beta_{k}\right) A u_{0}^{h} \stackrel{\text { def }}{=} v^{h} .
$$

From $A$-stability (8) it follows that $\beta>0$. Hence,

$$
u_{k}^{h}=J_{h \beta} v^{h} .
$$

Thus, using (i)-(iii), and writing $J_{\lambda} x-x=\lambda A_{\lambda} x$,

$$
\begin{aligned}
\left|u_{k}^{h}-u_{0}^{h}\right| & \leqslant\left|J_{h \beta} v^{h}-v^{h}\right|+\left|v^{h}-u_{0}^{h}\right| \\
& \leqslant h \beta\left|A_{h \beta} v^{h}\right|+h|\sigma(1)-\beta|\left|A u_{0}^{h}\right| \\
& \leqslant h \beta\left|A_{h \beta} v^{h}-A_{h \beta} u_{0}\right|+h \beta\left|A_{h \beta} u_{0}\right|+h|\sigma(1)-\beta|\left|A_{h} u_{0}\right| \\
& \leqslant\left|v^{h}-u_{0}\right|+h[2 \beta+|\sigma(1)|]\left|A u_{0}\right| \\
& \leqslant\left|u_{0}^{h}-u_{0}\right|+h|\sigma(1)-\beta|\left|A u_{0}\right|+h[2 \beta+|\sigma(1)|]\left|A u_{0}\right| \\
& \leqslant h\{1+3 \beta+2|\sigma(1)|\}\left|A u_{0}\right|=C\left|A u_{0}\right| h .
\end{aligned}
$$

From (36) we then also obtain $h \beta_{k}\left|A u_{k}^{h}\right| \leqslant C\left|A u_{0}\right| h$. Substituting these into (35) gives

$$
\left|u_{n+1}^{h}-u_{n}^{h}\right| \leqslant C\left|A u_{0}\right| h
$$


and, hence,

$$
\left|\rho(E) u_{n}^{h}\right| \leqslant C\left|A u_{0}\right| h \text { for all } n \geqslant 0 .
$$

This give us

$$
\left|\sigma(E) A u_{n}^{h}\right| \leqslant C\left|A u_{0}\right| \text { for all } n \geqslant 0
$$

But (8) and (9) imply that the roots of $\sigma(\zeta)$ lie strictly inside the unit circle and, therefore, (37) implies (34). Summarizing (30), (31), (33) and (34) we have

$$
\begin{aligned}
\delta\left|u_{N}^{h}-u_{\lambda, N}^{h}\right|^{2} & \leqslant C T\left|A u_{0}\right|^{2} h+\frac{\lambda}{2} h \sum_{0}^{N}\left|A u_{n}^{h}\right|^{2} \\
& \leqslant C T\left|A u_{0}\right|^{2}(h+\lambda), \quad N<T / h
\end{aligned}
$$

and since $\delta>0$,

$$
\left|u_{n}^{h}-u_{\lambda, n}^{h}\right| \leqslant C\left|A u_{0}\right| \sqrt{ }[T(h+\lambda)], \quad 0 \leqslant n<T / h ; h, \lambda>0 .
$$

Combining (25), (26) and (38) we thus have

$$
\begin{aligned}
\left|u(n h)-u_{n}^{h}\right| & \leqslant\left|u(n h)-u_{\lambda}(n h)\right|+\left|u_{\lambda}(n h)-u_{\lambda, n}^{h}\right|+\left|u_{\lambda, n}^{h}-u_{n}^{h}\right| \\
& \leqslant C\left|A u_{0}\right|\{\sqrt{ }(T \lambda)+h+T h \lambda+\sqrt{ }[T(h+\lambda)]\}
\end{aligned}
$$

which then implies (11) and completes the proof. Corollary 1 follows if in (39) we substitute $h^{2 / 3}$ for $\lambda=\lambda(h)$.

4. Proof of Theorem 3. We shall assume that $u_{0} \in D(A)$. The general case follows as in the proof of Theorem 2, since the bounds (19) and (21) are independent of $T$.

Let $c \in A^{-1} 0$ be such that $u(t) \rightarrow c$ (strongly) as $t \rightarrow \infty$. Put $v_{n}=c$ for all $n \geqslant 0$ and $u_{n}=u_{n+N}^{h}$. Then Theorem 1 gives us

$$
\left|u_{n}^{h}-c\right| \leqslant C\left\{\max _{0 \leqslant j<k}\left[\left|u_{N+j}^{h}-c\right|+h\left|A u_{N+j}^{h}\right|\right]\right\} \quad \text { for } n \geqslant N .
$$

Together with (34) this gives

$$
\left|u_{n}^{h}-c\right| \leqslant C \max _{0 \leqslant j<k}\left|u_{N+j}^{h}-c\right|+C h\left|A u_{0}\right| \text { for } n \geqslant N
$$

Fix $\epsilon>0$ and take $T<\infty$ so that

$$
|u(t)-c|<\epsilon \text { for } t \geqslant T
$$

Choose $h_{0}<1 / k$ such that, by Theorem 2,

$$
\left|u(n h)-u_{n}^{h}\right|<\epsilon \text { for } n h<T+2 \text { for } h<h_{0} .
$$


Put $N=[T / h]+1$. For $m h>T$ we then have

$$
\begin{aligned}
\left|u(m h)-u_{m}^{h}\right| & \leqslant|u(m h)-c|+\left|c-u_{m}^{h}\right| \\
& <\epsilon+C \max _{0<j<k}\left|u_{N+j}^{h}-c\right|+C h\left|A u_{0}\right| .
\end{aligned}
$$

But using (42) and (43), we get

$$
\left|u_{N+j}^{h}-c\right| \leqslant\left|u_{N+j}^{h}-u((N+j) h)\right|+|u((N+j) h)-c|<2 \epsilon
$$

and hence,

$$
\left|u(m h)-u_{m}^{h}\right|<(2 C+1) \epsilon+C h\left|A u_{0}\right|, \quad m h>T
$$

and the conclusion follows.

5. Proof of Theorem 4. For the trapezoidal rule we do not have a bound of the form (10); but we shall overcome this difficulty by considering a closely related method, the implicit midpoint rule

$$
h^{-1}\left(\hat{u}_{n+1}^{h}-\hat{u}_{n}^{h}\right)+A\left(\frac{\hat{u}_{n+1}^{h}+\hat{u}_{n}^{h}}{2}\right)=0 \text { for } n \geqslant 0 .
$$

Existence and uniqueness of a solution $\left\{\hat{u}_{n}^{h}\right\}$ is obtained as follows: Write (45) in the form

$$
v_{n}+\frac{h}{2} A v_{n}=\hat{u}_{n}^{h}
$$

with $\hat{u}_{n}^{h}$ given and $v_{n}\left(=1 / 2\left(\hat{u}_{n+1}^{h}+\hat{u}_{n}^{h}\right)\right)$ unknown. But then $v_{n}=J_{h / 2} \hat{u}_{n}^{h} \in D(A)$ and the process may be continued. Further,

$$
v_{n+1}-v_{n}=\frac{\hat{u}_{n+2}^{h}-\hat{u}_{n}^{h}}{2}=-\frac{h}{2}\left(A v_{n+1}+A v_{n}\right)
$$

so that $\left\{v_{n}\right\}$ satisfies (15) with the initial value $v_{0}=J_{h / 2} \hat{u}_{0}^{h} \in D(A)$. On the other hand, (15) has a unique solution $\left\{u_{n}^{h}\right\} \subset D(A)$ for all $u_{0}^{h} \in D(A)$ since

$$
u_{n+1}^{h}=J_{h / 2}\left(u_{n}^{h}+\frac{h}{2} A u_{n}^{h}\right) \in D(A) \text { for all } n \geqslant 0 .
$$

Hence we have reduced our problem to showing that, with $\hat{u}_{0}^{h}=u_{0}$,

$$
\sup _{0 \leqslant n<T / h}\left|u(n h)-\hat{u}_{n}^{h}\right| \rightarrow 0 \quad \text { as } h \downarrow 0 ;
$$

and that if (13) holds, then

$$
\sup _{n \geqslant 0}\left|u(n h)-\hat{u}_{n}^{h}\right| \rightarrow 0 \quad \text { as } h \downarrow 0 .
$$


Convergence for

$$
v_{n}=\left(\hat{u}_{n+1}^{h}+\hat{u}_{n}^{h}\right) / 2
$$

will follow since $u(t)$ is uniformly continuous on compact sets $[0, T]$ and, assuming (13), on the half line $t \geqslant 0$.

For notational simplicity, consider the solutions $\left\{x_{n}\right\},\left\{y_{n}\right\}$ of the equations

$$
\begin{aligned}
& h^{-1}\left(x_{n+1}-x_{n}\right)+A\left(\frac{x_{n+1}+x_{n}}{2}\right)=0, \\
& h^{-1}\left(y_{n+1}-y_{n}\right)+A\left(\frac{y_{n+1}+y_{n}}{2}\right)=0 .
\end{aligned}
$$

Forming the difference of (47) and (48), multiplying it by $1 / 2\left(x_{n+1}-y_{n+1}-x_{n}+y_{n}\right)$, and using the monotonicity of $A$ yields

$$
\left|x_{n+1}-y_{n+1}\right| \leqslant\left|x_{n}-y_{n}\right|, \quad n \geqslant 0 .
$$

Applying (49) with $y_{n}=x_{n+1}$ gives

$$
\left|A\left(\frac{x_{n+1}+x_{n}}{2}\right)\right| \leqslant h^{-1}\left|x_{1}-x_{0}\right|=\left|A_{h / 2} x_{0}\right| \leqslant\left|A x_{0}\right| .
$$

Denote by $x_{\lambda, n}$ the solution of (47) with $A$ replaced by $A_{\lambda}$ and $x_{\lambda, 0}=x_{0}$. Multiplying

$$
x_{n+1}-x_{\lambda, n+1}-x_{n}+x_{\lambda, n}+h\left\{A\left(\frac{x_{n+1}+x_{n}}{2}\right)-A_{\lambda}\left(\frac{x_{\lambda, n+1}+x_{\lambda, n}}{2}\right)\right\}=0
$$

by $1 / 2\left\{x_{n+1}-x_{\lambda, n+1}+x_{n}-x_{\lambda, n}\right\}$, we get, using (32), and (50),

$$
\left|x_{n}-x_{\lambda, n}\right| \leqslant\left|A x_{0}\right| \sqrt{ }(\lambda n h) \text { for } n \geqslant 0
$$

that is,

$$
\left|\hat{u}_{n}^{h}-\hat{u}_{\lambda, n}^{h}\right| \leqslant\left|A u_{0}\right| \sqrt{ }(\lambda n h) \text { for } n \geqslant 0 ; \lambda, h>0 .
$$

Since the implicit midpoint rule is a convergent method (for Lipschitz-continuous problems on compact intervals $[0, T]$ ) the proof of the reduced problem can now be completed as in the earlier chapters, using (51), and (49) or

$$
\left|\hat{u}_{n+1}^{h}-\hat{v}_{n+1}^{h}\right| \leqslant\left|\hat{u}_{n}^{h}-\hat{v}_{n}^{h}\right|
$$

in place of (38) and (10).

Remark. The implicit midpoint rule is the one-leg method associated with the trapezoidal rule, [6]. A generalization of Theorem 4 to all $G$-stable [6] methods is straightforward but since the trapezoidal rule is the only practically important method which does not satisfy (9) we do not want to introduce the machinery needed. 
However, if the conjecture that $A$-stability implies $G$-stability (see [7]) is correct, then some parts of the proof of Theorem 2 could be simplified. The analogue of Theorem 1 for $G$-stable one-leg methods has been given in [11], with a more direct proof than in $[10]$.

6. Proof of Theorem 5. Put $a=-1 / r$, where $r$ is given by (16). Assume that $\left(I+a h A_{\lambda}\right)^{-1}$ is Lipschitz-continuous with constant $L=L(\lambda, h)<\infty$ and that $A_{\lambda}\left(I+a h A_{\lambda}\right)^{-1}-\alpha I$ is maximally monotone with $\alpha=\alpha(\lambda, h)>-\infty$. Then we obtain, using line (3.23) in [12], that for $C, b>0$ depending only on $(\rho, \sigma)$,

$$
\left|u_{\lambda, n}^{h}-u_{\lambda}(n h)\right| \leqslant C L(\lambda, h)(1-|\alpha(\lambda, h)| h b)^{-(1+T) / 2 h}
$$

$$
\cdot\left\{h \sum_{n=k}^{T / h}\left|\tau_{\lambda, n}^{h}\right|+\max _{0 \leqslant j<k}\left[\left|u_{0}-u_{\lambda}(j h)\right|+h\left|A_{\lambda} u_{0}-A_{\lambda} u_{\lambda}(j h)\right|\right]\right\} .
$$

From (23) we obtain $\left|\tau_{\lambda, n}^{h}\right| \leqslant C(h / \lambda)\left|A u_{0}\right|$ and, therefore,

$$
h \sum_{n=k}^{T / h}\left|\tau_{\lambda, n}^{h}\right| \leqslant C T\left|A u_{0}\right|(h / \lambda)
$$

Since $\left|A_{\lambda} u_{\lambda}(t)\right|$ is nonincreasing in $t$, we also have

$$
\begin{aligned}
\max _{0 \leqslant j<k}\left[\left|u_{0}-u_{\lambda}(j h)\right|+h\left|A_{\lambda} u_{0}-A_{\lambda} u_{\lambda}(j h)\right|\right] \\
\quad \leqslant(k-1) h\left|A_{\lambda} u_{0}\right|+h\left|A_{\lambda} u_{0}\right|+h\left|A_{\lambda} u_{0}\right| \leqslant(k+1) h\left|A u_{0}\right| .
\end{aligned}
$$

Thus, (53) is of the form

$$
\left|u_{\lambda}(n h)-u_{\lambda, n}^{h}\right| \leqslant C L(\lambda, h)\{1-|\alpha(\lambda, h)| b h\}^{-(1+T) / 2 h}\left|A u_{0}\right|\left\{h+\frac{h T}{\lambda}\right\}
$$

Since $A_{\lambda}$ is Lipschitz-continuous with constant $1 / \lambda$, we have $L(\lambda, h) \leqslant(1-|a| h / \lambda)^{-1}$ $\rightarrow 1$ if $h / \lambda \rightarrow 0$. To estimate $\alpha(\lambda, h)$ from below, put $x=\left(I+a h A_{\lambda}\right)^{-1} u$ and $y=\left(I+a h A_{\lambda}\right)^{-1} v$. Then

$$
\begin{aligned}
& \left(A_{\lambda}\left(I+a h A_{\lambda}\right)^{-1} u-A_{\lambda}\left(I+a h A_{\lambda}\right)^{-1} v, u-v\right) \\
& \quad=\left(A_{\lambda} x-A_{\lambda} y, x-y\right)+a h\left(A_{\lambda} x-A_{\lambda} y, A_{\lambda} x-A_{\lambda} y\right) \\
& \quad \geqslant a h\left|A_{\lambda} x-A_{\lambda} y\right|^{2} \geqslant\left(a h / \lambda^{2}\right)|x-y|^{2} \geqslant a h(L(\lambda, h) / \lambda)^{2}|u-v|^{2},
\end{aligned}
$$

so that

$$
|\alpha(\lambda, h)| \leqslant|a| h\left(\frac{L(\lambda, h)}{\lambda}\right)^{2} \leqslant|a| / c
$$

if $\lambda \downarrow 0$ with $\lambda^{2} \geqslant c h$; and we conclude that $\{1-|\alpha(\lambda, h)| b h\}^{-(1 / 2 h)(1+T)}$ remains bounded for $\lambda$ small, $\lambda<\lambda_{0}$ say. 
Summarizing (54) and (26), we have for $n h<T$,

$$
\begin{aligned}
\left|u(n h)-u_{\lambda, n}^{h}\right| & \leqslant\left|u(n h)-u_{\lambda}(n h)\right|+\left|u_{\lambda}(n h)-u_{\lambda, n}^{h}\right| \\
& \leqslant C\left(\lambda_{0}, T\right)\left|A u_{0}\right|\left\{\lambda^{1 / 2}+h+h / \lambda\right\}
\end{aligned}
$$

which completes the proof.

Institute of Mathematics

Helsinki University of Technology

SF-02150 Espoo 15, Finland

1. H. BREZIS, Opérateurs Maximaux Monotones, North-Holland, Amsterdam, 1973.

2. H. BREZIS \& A. PAZY, "Semi groups of nonlinear contractions on convex sets," $J$. Functional Analysis, v. 6, 1970, pp. 237-281.

3. R. BRUCK, "Asymptotic convergence of nonlinear contraction semi groups in Hilbert space," J. Functional Analysis, v. 18, 1975, pp. 15-26.

4. M. CRANDALL \& T. LIGGETT, "Generation of semigroups of nonlinear transformations on general Banach spaces," Amer. J. Math., v. 93, 1971, pp. 265-298.

5. G. DAHLQUIST, "A special stability problem for linear multistep methods," BIT, v. 3, 1963, pp. $27-43$.

6. G. DAHLQUIST, Error Analysis for a Class of Methods for Stiff Non-Linear Initial Value Problems, Lecture Notes in Math., vol. 506, Springer-Verlag, Berlin and New York, 1976, pp. 60-74.

7. G. DAHLQUIST, On the Relation of G-Stability to Other Stability Concepts for Linear Multistep Methods, Report TRITA-NA-7618, Dept. of Comput. Sci., Royal Inst. of Tech., 1976.

8. J. KAČUR, "The Rothe method and nonlinear parabolic equations of arbitrary order," Theory of Nonlinear Operators (Proc. Summer-school held in Oct. 1972 at Neuendorf), AkademieVerlag, Berlin, 1974, pp. 125-131.

9. N. KENMOCHI \& S. OHARU, "Difference approximation of nonlinear evolution equations and semigroups of nonlinear operators," Publ. Res. Inst. Math. Sci., v. 10, 1974, pp. 147207. $79-84$.

10. O. NEVANLINNA, "On error bounds for $G$-stable methods," $B I T$, v. 16, 1976, pp.

11. O. NEVANLINNA, On Multistep Methods for Nonlinear Initial Value Problems with an Application to Minimization of Convex Functionals, Report HTKK-MAT-A76, Inst. of Math., Helsinki Univ. of Tech., 1976.

12. O. NEVANLINNA, "On the numerical integration of nonlinear initial value problems by linear multistep methods," BIT, v. 17, 1977, pp. 58-71.

13. T. TAKAHASHI, "Convergence of difference approximation of nonlinear evolution equations and generation of semigroups," J. Math. Soc. Japan, v. 28, 1976, pp. 96-113. 